
\title{
28 Research Square \\ The Effects of Antithrombotic Therapy on Head Trauma and its Management
}

\section{Takao Koiso ( $\nabla$ s0201534@hotmail.co.jp )}

Hitachi General Hospital

\section{Yoji Komatsu}

Hitachi General Hospital

Masayuki Goto

Hitachi General Hospital

\section{Toshitsugu Terakado}

Hitachi General Hospital

\section{Yuji Matsumaru}

University of Tsukuba

\section{Eichi Ishikawa}

University of Tsukuba

\section{Research Article}

Keywords: antithrombotic drugs, traumatic head injury, restart

Posted Date: March 18th, 2021

DOI: https://doi.org/10.21203/rs.3.rs-311483/v1

License: (c) (i) This work is licensed under a Creative Commons Attribution 4.0 International License.

Read Full License 


\section{Abstract}

To examine the effects of antithrombotics for head trauma, 393 consecutive patients were enrolled. The patients were divided into those that were (group $A, n=117$ ) and were not (group $B, n=276$ ) taking antithrombotics, and the groups' outcomes were compared. To identify factors that affected functional independence in group A, clinical factors were compared between the patients that exhibited mRS of 0-2 and 3-6 at discharge. Furthermore, to assess the optimal time to restart antithrombotics, cases in which rebleeding occurred after antithrombotics were restarted or thromboembolic events occurred were extracted. The ratio of mRS 3-6 and death within 30 days were significantly higher in group A than in group B. Multivariate analysis of group A revealed that being aged $\geq 70$, not receiving antiplatelet therapy, and intracranial hematoma enlargement were poor prognostic factors. Five patients in group A experienced ischemic strokes within 30 days. In 3 of these patients, the ischemic strokes occurred before antithrombotics were restarted. Rebleeding occurred in two cases after anticoagulants restarted within 48 hours. Patients taking antithrombotics are at high risk of poor prognosis after head trauma. To prevent thromboembolic events, the active resumption of antithrombotics after 48 hours is desirable if hemostasis has been achieved.

\section{Introduction}

As the Japanese population ages, the number of head trauma patients that are taking antithrombotic drugs is increasing. The mortality rate of spontaneous intracerebral hemorrhage (ICH) at 90 days was higher in users of antithrombotic drugs than in non-users $(32.3 \% \text { vs. } 23.0 \%, p<0.001)^{1}$. On the other hand, the reinstitution of anticoagulation therapy after spontaneous $\mathrm{ICH}$ associated with a lower risk of thromboembolic complications and a similar risk of ICH recurrence ${ }^{2}$. There have been few reports about head trauma patients who are taking antithrombotic drugs, and the influence of such drugs on outcomes and the optimal time to restart antithrombotic therapy are disputed. In this study, we retrospectively reviewed the medical records of head trauma patients that were being treated with antithrombotic drugs to determine the effects of such drug treatment on outcomes and the optimal time to restart antithrombotic therapy.

\section{Methods}

\section{Ethical Approval}

All procedures performed in this study was approved by the local ethics committee of the Hitachi General Hospital (Research Ethics Committee of Corporate Hospital Group, Hitachi, Ltd: \#2019-71), and complied with the conditions laid out by the Declaration of Helsinki. The opt-out consent was used for patients and the requirement to obtain informed consent was waived by the ethics committee.

\section{Patient Population}


The dataset consisted of 727 consecutive head trauma patients who were treated during the 7-year period between April 2012 and March 2019. After excluding 50 patients who were aged under 16 years old, 206 patients with chronic subdural hematomas, 62 patients without intracranial hematomas, 4 patients with spontaneous $\mathrm{ICH}, 9$ patients whose modified Rankin Scale score (mRS) was $\geq 3$ before the trauma, and 3 patients for whom detailed information was not available, 393 patients remained.

All head trauma patients that are admitted to Hitachi General Hospital undergo an initial head computed tomography (CT) scan within 60 minutes of arrival and a $2^{\text {nd }}$ CT scan within 24 hours of admission. Of the 393 patients mentioned above, 46 did not undergo a $2^{\text {nd }}$ CT scan, as they required emergency surgery. Twenty further patients did not undergo a $2^{\text {nd }}$ CT scan because they died or had very small hematomas. Thus, repeated CT scans were performed in 327 patients.

\section{Imaging protocol}

All CT scans were performed using a SCENARIA CT scanner (Hitachi Medical Corporation, Hitachi, Japan) with a 2.5-mm slice thickness. The existence and enlargement of intracranial hematomas were evaluated by the first author. Intracerebral hematoma volume was calculated using the " $\mathrm{ABC} / 2$ " formula 3 . "Enlargement" was defined as when the hematoma volume increased by more than $25 \%$. In the case of epidural hematomas, subdural hematomas, or subarachnoid hemorrhaging, this was judged based on the thickness of the thickest part.

\section{Clinical Outcomes}

The primary endpoint was global disability, which was assessed based on the mRS at discharge. The secondary endpoints were enlargement of the intracranial hematoma within 72 hours, all-cause mortality within 30 days, and in-hospital neurological death. Neurological death was defined as death caused by any intracranial disease that was identified by neurosurgeons.

To determine the effects of antithrombotic drugs on outcomes, we compared clinical factors and outcomes between the patients that were and were not treated with such drugs. To determine the clinical factors that affected the independence of head trauma patients treated with antithrombotic drugs, we also divided the patients that were treated with antithrombotic drugs into two groups according to their mRS at discharge (0-2 or 3-6) and compared clinical factors between the groups. The optimal time to restart antithrombotic drug treatment was investigated by examining the cases in which rebleeding occurred after antithrombotic drug treatment was restarted or in-hospital thromboembolic events (ischemic stroke and/or myocardial infarction) occurred.

\section{Statistical Analysis}

Summary statistics for the examined variables are presented (frequencies and percentages for categorical data and medians and interquartile ranges for continuous data). Fisher's exact test was used for analyzing categorical data, and the Wilcoxon rank sum test was used for analyzing continuous data. 
Multivariate logistic regression analysis was performed to determine the clinical factors on admission that affected outcomes.

All comparisons were planned, and all tests were 2-sided. P-values of $<0.05$ were considered to be statistically significant. All statistical analyses were performed using JMP (Japanese version 12 for Windows; SAS Institute Inc., Cary, NC, USA).

\section{Results}

Table 1 summarizes the clinical characteristics of the whole study population and the two groups; i.e., the patients that were (group A, 117 patients) and were not (group B, 276 patients) treated with antithrombotic drugs. In group A, 62 patients (53.0\%) were using antiplatelets alone, 38 patients (32.5\%) were using anticoagulants alone, and 17 patients (14.5\%) were using both types of drugs. The most common reason for antiplatelet use was a cardiovascular event (35/66 (43.2\%); i.e., myocardial infarction) followed by a cerebrovascular event (25/66 (30.9\%)). The most common reasons for using anticoagulants were atrial fibrillation or cardiac valve surgery $(47 / 53(85.5 \%))$. The reasons for antithrombotic drug use were unknown in 13 patients who were using antiplatelets and 2 patients who were using anticoagulants. The mean age of group $A$ was significantly higher than that of group $B$ (79.0 vs. $71.0, p<0.0001)$. ICH was found in $42(35.9 \%)$ patients in group $A$ and $133(48.2 \%)$ patients in group $B$ $(p=0.0268)$. Eighteen $(15.4 \%)$ patients in group $A$ and $79(28.6 \%)$ patients in group $B$ had extracranial injuries $(p=0.0049)$.

Sixty-six (56.4\%) patients in group A and 117 (42.4\%) patients in group B had mRS of 3-6 at discharge $(p=0.0113)$. Thirty $(25.6 \%)$ patients in group $A$ and $45(16.3 \%)$ patients in group $B$ died within 30 days of their injuries occurring $(p=0.0356)$. As shown in Table 2, $66(56.4 \%)$ patients in group $A$ and $117(42.4 \%)$ patients in group $B$ had mRS of 3-6 at discharge $(p=0.0113)$. However, the incidence of hematoma enlargement did not differ significantly between groups A and B. The incidence of neurological death also did not differ significantly between the groups.

Subanalyses revealed that hematoma enlargement occurred in 24 of $50(48.0 \%)$ patients that were being treated with anticoagulant drugs and in 93 of 301 (30.9\%) patients that were not being treated with such drugs. Hematoma enlargement also occurred in 87 of 157 (55.4\%) patients in whom ICH was detected on the $1^{\text {st }} \mathrm{CT}$ scan and in 30 of $194(15.5 \%)$ patients in which ICH was not detected on the $1^{\text {st }} \mathrm{CT}$ scan. Anticoagulant drug use and the presence of $\mathrm{ICH}$ on the $1^{\text {st }} \mathrm{CT}$ exhibited significant associations with enlargement $(p=0.0229$ and $p<0.0001$, respectively). The use of antiplatelet drugs, however, was not associated with hematoma enlargement.

The duration of the period from admission to hematoma enlargement did not differ significantly between groups A and B ( 4 vs. 3 hours, $p=0.4613$ ). In almost all cases, hematoma enlargement occurred within 24 hours, regardless of whether the patient was taking antithrombotic drugs. 
Among the patients taking antithrombotic drugs, the univariate analyses revealed that age, antiplatelet therapy, the presence of an intracerebral hematoma, and intracranial hematoma enlargement occurring within 72 hours were significant predictors of outcomes (Table 3). The multivariate analysis revealed that being aged $\geq 70$ (odds ratio [OR]: $7.25,95 \%$ confidence interval [95\% $\mathrm{Cl}]: 1.65-43.01, p=0.0076$ ), the absence of antiplatelet therapy (OR: $2.73,95 \% \mathrm{Cl} 1.03-7.87, \mathrm{p}=0.0440)$, and intracranial hematoma enlargement (OR: $3.11,95 \% \mathrm{Cl}: 1.03-10.20, p=0.0447)$ were significant unfavorable prognostic indicators.

Among the patients being treated with antithrombotic drugs, ischemic strokes occurred during the period from day 1 to day 31 after the injury in 5 cases. In 3 of the 5 cases, the ischemic strokes occurred before antithrombotic drug treatment was restarted. In a case in which 4-factor prothrombin complex concentrate (4F-PCC) was administered when the patient was injured, an ischemic stroke occurred on day 1. A total of 9 patients were given $4 \mathrm{~F}-\mathrm{PCC}$, and 2 of them (22.2\%) had ischemic strokes after the administration of the drug. On the other hand, 20 patients were taking vitamin $\mathrm{K}$ antagonists (VKA), and only one of them (5\%) experienced an ischemic stroke. There were no cases of myocardial infarction in this study.

Antiplatelet drug treatment was restarted in 21 of 79 cases (26.6\%), and anticoagulant treatment was restarted in 35 of 55 cases (63.6\%). Rebleeding occurred in 2 cases in which anticoagulant treatment was restarted within 2 days of the injury, and these events influenced the patients' outcomes.

\section{Discussion}

This study investigated the outcomes of head trauma patients that were taking antithrombotic drugs. The results showed that the patients that were taking antithrombotic drugs had poor outcomes despite exhibiting lower frequencies of $\mathrm{ICH}$ and extracranial injuries than the patients that were not taking antithrombotic drugs. This suggests that antithrombotic drugs can cause poor outcomes even in cases of lower-energy trauma. A previous retrospective cohort study suggested that antiplatelet therapy could be a risk factor for hematoma enlargement and poor outcomes after mild head trauma ${ }^{4}$. Among the patients using antiplatelet drugs, the risk of hematoma enlargement was two-fold higher and the risk of a poor outcome was $50 \%$ higher (relative risk: $1.58,95 \% \mathrm{Cl}$ : 1.28-1.95). Anticoagulant drug use was also reported to be related to poor outcomes ${ }^{5}$. The adjusted OR for 30-day mortality of VKA users was 8.3 (95\%Cl: 2.0-34.8).

In this study, being aged $\geq 70$, the absence of antiplatelet drug treatment, and the intracranial hematoma enlargement occurring within 72 hours were identified as predictors of poor outcomes in the patients being treated with antithrombotic drugs. Subanalyses revealed that hematoma enlargement was related to anticoagulant drug use, but not antiplatelet drug use. Some studies of spontaneous ICH have shown that hematoma enlargement was associated with poor outcomes ${ }^{6-13}$. In addition, it was also reported that in cases of $\mathrm{ICH}$ hematoma volume was related to anticoagulant drug use, but was not related to antiplatelet drug use ${ }^{14}$. A retrospective study of head trauma also revealed that that VKA use alone, but not antiplatelet drug use alone or direct oral anticoagulant use, was a significant predictor of the 
progression of ICH compared with non-use of these drugs (OR: $5.30,95 \% \mathrm{Cl}: 2.48-11.31)^{15}$. Therefore, head trauma patients that are taking anticoagulant drugs, especially VKA, could have a poor prognosis.

Almost all cases of hematoma enlargement occurred within 24 hours of the initial injury, regardless of whether the patients were using antithrombotic drugs. A retrospective review of 419 spontaneous ICH reported that hematoma enlargement often occurred within 6 hours of onset ${ }^{10}$. In a retrospective review of spontaneous $\mathrm{ICH}$, two thirds of cases of hematoma enlargement occurred within 24 hours of onset ${ }^{16}$. Moreover, a case study of mild head trauma reported that $87 \%$ of cases of delayed neurological deterioration occurred within 24 hours ${ }^{17}$. On the other hand, achieving hemostasis takes longer than normal in patients taking antithrombotic drugs ${ }^{18}$. In a prospective cohort study of ICH patients that were taking VKA, 3 of 7 patients exhibited hematoma enlargement within 24 hours $^{19}$. The time from the injury to hemostasis being achieved could not be examined in this study, but close follow-up, involving neurological examinations and CT, for at least within 24 hours are considered important for ensuring that treatment is timed appropriately.

The appropriate use of reversal agents seems to be important for preventing the expansion of hematomas and improving outcomes. Two randomized controlled trials of 4F-PCC vs. vitamin $\mathrm{K}$ or plasma for cases of spontaneous ICH involving patients that were taking VKA showed that 4F-PCC had a superior ability to rapidly reduce the international normalized ratio ${ }^{20,21}$. In addition, 4F-PCC reduced the frequencies of hematoma enlargement and hematoma-related early death ${ }^{22}$. As the number of patients treated with reversal agents has increased, however, the frequency of thromboembolic events has also risen. In this study, ischemic strokes occurred in 2 of the 9 cases in which 4F-PCC was used. One of these patients suffered a cerebral infarction within 24 hours. In a previous case report, an ischemic stroke occurred on the day after warfarin reversal with 4F-PCC for surgery ${ }^{23}$. To prevent thromboembolic events, the early resumption of antithrombotic drug treatment is necessary. In a retrospective study of chronic subdural hematomas, the early resumption of antithrombotic drug treatment was recommended because many thromboembolic events occurred within one month of drug withdrawal ${ }^{24}$. In a meta-analysis of spontaneous $\mathrm{ICH}$, the resumption of anticoagulant treatment between days 10 and 39 after onset produced good results ${ }^{2}$. In the present study, there were 2 cases of rebleeding after antithrombotic drug therapy was restarted within 2 days of admission. Therefore, the early resumption of antithrombotic drug treatment is considered beneficial when hemostasis has been confirmed on CT after more than 48 hours.

\section{Limitations of This Study}

This study was retrospective, and hence, background factor variability was its greatest limitation. Lowenergy trauma is probably more common among patients taking antithrombotic drugs, which may explain why no correlation was detected between antithrombotic drug use and hematoma enlargement. A prospective cohort study is needed to provide more accurate data about the outcomes of head trauma among patients taking antithrombotic drugs. 
In conclusion, patients that are taking antithrombotic drugs are at high risk of a poor prognosis after head trauma, even after low-energy head trauma. Among such patients, the prognosis of those that exhibit acute hematoma enlargement is poor, and active hemostasis, such as using neutralizers, is important. In order to prevent thromboembolic events, the active resumption of antithrombotic drugs is desirable in cases in which hemostasis has been confirmed after more than 48 hours.

\section{Declarations}

\section{Data availability}

The datasets generated during and/or analysed during the current study are available from the corresponding author on reasonable request.

\section{Author Contributions Statement}

TK and YK were involved in the study conception and design, the data acquisition and analysis, and the writing and drafting of the manuscript. MG and TT were involved in the study conception and data acquisition. YM and El were involved in the study conception and the drafting of the manuscript. The authorship requirements have been met by all of the authors. All of the authors have read and approved the final version of the manuscript.

\section{Competing interests}

The authors declare that they have no potential conflicts of interest with respect to the research, authorship, and/or publication of this article.

\section{References}

1. Gulati, S. et al. Risk of intracranial hemorrhage (RICH) in users of oral antithrombotic drugs: Nationwide pharmacoepidemiological study. PloS one13, e0202575 (2018).

2. Murthy, S.B. et al. Restarting Anticoagulant Therapy After Intracranial Hemorrhage: A Systematic Review and Meta-Analysis. Stroke48, 1594-600 (2017)

3. Kothari, R.U. et al. The ABCs of measuring intracerebral hemorrhage volumes. Stroke27, 1304-5 (1996)

4. Fabbri, A. et al. Antiplatelet therapy and the outcome of subjects with intracranial injury: the Italian SIMEU study. Critical care17, R53 (2013)

5. Narum, S., Brørs, O., Stokland, O. \& Kringen, M.K. Mortality among head trauma patients taking preinjury antithrombotic agents: a retrospective cohort analysis from a Level 1 trauma centre. $B M C$ emergency medicine16, 29 (2016)

6. Broderick, J.P., Brott, T.G., Duldner, J.E., Tomsick, T. \& Huster, G. Volume of intracerebral hemorrhage. A powerful and easy-to-use predictor of 30-day mortality. Stroke24, 987-93 (1993) 
7. Brott, T. et al. Early hemorrhage growth in patients with intracerebral hemorrhage. Stroke28, 1-5 (1997)

8. Daverat, P., Castel, J.P., Dartigues, J.F. \& Orgogozo, J.M. Death and functional outcome after spontaneous intracerebral hemorrhage. A prospective study of 166 cases using multivariate analysis. Stroke22, 1-6 (1991)

9. Fujii, Y., Takeuchi, S., Sasaki, O., Minakawa, T. \& Tanaka, R. Multivariate analysis of predictors of hematoma enlargement in spontaneous intracerebral hemorrhage. Stroke29, 1160-6 (1998)

10. Fujii, Y., et al. Hematoma enlargement in spontaneous intracerebral hemorrhage. JNS 80, $51-7$ (1994)

11. Kazui, S., Minematsu, K., Yamamoto, H., Sawada, T. \& Yamaguchi, T. Predisposing factors to enlargement of spontaneous intracerebral hematoma. Stroke28, 2370-5 (1997)

12. Sorimachi, T., Fujii, Y., Morita, K. \& Tanaka, R. Predictors of hematoma enlargement in patients with intracerebral hemorrhage treated with rapid administration of antifibrinolytic agents and strict blood pressure control. JNS106, 250-4 (2007)

13. Zazulia, A.R., Diringer, M.N., Derdeyn, C.P. \& Powers, W.J. Progression of mass effect after intracerebral hemorrhage. Stroke30, 1167-73 (1999)

14. Foerch, C., Sitzer, M., Steinmetz, H. \& Neumann-Haefelin, T. Pretreatment with antiplatelet agents is not independently associated with unfavorable outcome in intracerebral hemorrhage. Stroke37, 2165-7 (2006)

15. Scotti, P. et al. Antithrombotic agents and traumatic brain injury in the elderly population: hemorrhage patterns and outcomes. JNS5, 1-10 (2019)

16. Lim, J.K. et al. Multivariate analysis of risk factors of hematoma expansion in spontaneous intracerebral hemorrhage. Surgical neurology69, 40-5 (2008)

17. Choudhry, O.J., Prestigiacomo, C.J., Gala, N., Slasky, S. \& Sifri, Z.C. Delayed neurological deterioration after mild head injury: cause, temporal course, and outcomes. Neurosurgery73, 753-60 (2013)

18. Lim-Hing, K. \& Rincon, F. Secondary Hematoma Expansion and Perihemorrhagic Edema after Intracerebral Hemorrhage: From Bench Work to Practical Aspects. Frontiers in neurology8, 74 (2017)

19. Flibotte, J.J., Hagan, N., O'Donnell, J., Greenberg, S.M. \& Rosand, J. Warfarin, hematoma expansion, and outcome of intracerebral hemorrhage. Neurology63, 1059-64 (2004)

20. Goldstein, J.N. et al. Four-factor prothrombin complex concentrate versus plasma for rapid vitamin K antagonist reversal in patients needing urgent surgical or invasive interventions: a phase $3 \mathrm{~b}$, openlabel, non-inferiority, randomised trial. Lancet385, 2077-87 (2015)

21. Sarode, R. et al. Efficacy and safety of a 4-factor prothrombin complex concentrate in patients on vitamin $\mathrm{K}$ antagonists presenting with major bleeding: a randomized, plasma-controlled, phase IIIb study. Circulation128, 1234-43 (2013)

22. Steiner, T. et al. Fresh frozen plasma versus prothrombin complex concentrate in patients with intracranial haemorrhage related to vitamin $\mathrm{K}$ antagonists $(\mathrm{INCH})$ : a randomised trial. The Lancet Neurology15, 566-73 (2016) 
23. Carson, L. \& Price, J.E. Ischemic Stroke Symptoms After Warfarin Reversal With 4-Factor Prothrombin Complex Concentrate Case Report. Hospital pharmacy55, 69-71 (2020)

24. Fornebo, I. et al. Role of antithrombotic therapy in the risk of hematoma recurrence and thromboembolism after chronic subdural hematoma evacuation: a population-based consecutive cohort study. Acta neurochirurgica159, 2045-52 (2017)

\section{Tables}

\begin{tabular}{|c|c|c|c|c|}
\hline & Total & Antithromboti & drug treatment & p-value \\
\hline & & Yes (group A) & No (group B) & \\
\hline No. of patients & 393 & 117 & 276 & \\
\hline Age, yrs & & & & \\
\hline Median & 75.0 & 79.0 & 71.0 & $<0.0001$ \\
\hline IQR & $64.0-81.0$ & 75.5-83.5 & $60.3-80.0$ & \\
\hline Sex & & & & \\
\hline Female & 129 (32.8\%) & 46 (39.3\%) & 83 (30.1\%) & 0.0791 \\
\hline Emergency ope & & & & \\
\hline Yes & 85 (21.6\%) & 29 (24.8\%) & 56 (20.3\%) & 0.3491 \\
\hline Intracerebral h & & & & \\
\hline Yes & 175 (44.5\%) & 42 (35.9\%) & $133(48.2 \%)$ & 0.0268 \\
\hline PT-INR & & & & \\
\hline Median & 0.98 & 1.07 & 0.97 & $<0.0001$ \\
\hline IQR & $0.92-1.07$ & $0.95-2.00$ & $0.91-1.03$ & \\
\hline APTT & & & & \\
\hline Median & 29.3 & 30.9 & 28.3 & $<0.0001$ \\
\hline IQR & $26.8-31.9$ & $28.1-34.9$ & $26.3-31.0$ & \\
\hline Extracranial inj & & & & \\
\hline Yes & 97 (24.7\%) & 18 (15.4\%) & 79 (28.6\%) & 0.0049 \\
\hline AND & 27 (6.9\%) & 11 (9.4\%) & $16(5.8 \%)$ & 0.1977 \\
\hline
\end{tabular}

Table 1 Summary of the clinical characteristics of 393 head trauma patients

AND: allow natural death, APTT: activated partial thromboplastin, IQR: interquartile range, PT-INR: prothrombin time (international normalized method) 


\begin{tabular}{|c|c|c|c|c|}
\hline & \multirow[t]{2}{*}{ Total } & \multicolumn{2}{|c|}{ Antithrombotic drug treatment } & \multirow[t]{2}{*}{ p-value } \\
\hline & & Yes (group A) & No (group B) & \\
\hline No. of patients & 393 & 117 & 276 & \\
\hline \multicolumn{5}{|c|}{ Enlargement of intracranial hematoma $<72 \mathrm{hrs}$} \\
\hline Yes & $117(33.3 \%)$ & $35(32.4 \%)$ & $83(33.1 \%)$ & 0.8070 \\
\hline \multicolumn{5}{|c|}{ Time from admission to hematoma enlargement, hrs } \\
\hline Median & 3 & 4 & 3 & 0.4613 \\
\hline IQR & $2-14$ & 3-14 & $2-14$ & \\
\hline mRS of 3-6 @discharge & $183(46.6 \%)$ & $66(56.4 \%)$ & $117(42.4 \%)$ & 0.0113 \\
\hline Death within 30 days & $75(19.1 \%)$ & $30(25.6 \%)$ & $45(16.3 \%)$ & 0.0356 \\
\hline Neurological death & $56(75.7 \%)$ & $20(71.4 \%)$ & 36 (78.3\%) & 0.5809 \\
\hline
\end{tabular}

Table 2 Summary of the outcomes of 393 head trauma patients IQR: interquartile range, mRS: modified Rankin scale score 


\begin{tabular}{|c|c|c|c|c|c|c|}
\hline & \multicolumn{2}{|c|}{ Outcome at discharge } & \multicolumn{3}{|c|}{ Univariate } & Multivariate \\
\hline & $\begin{array}{l}\text { mRS } \\
\text { (Good) }\end{array}$ & $\begin{array}{l}\mathrm{mRS} 3-6 \\
\text { (Poor) }\end{array}$ & $\begin{array}{l}\text { OR } \\
(95 \% C I)\end{array}$ & $\begin{array}{l}\mathrm{p}- \\
\text { value }\end{array}$ & $\begin{array}{l}\mathrm{OR} \\
(95 \% \mathrm{CI})\end{array}$ & $\begin{array}{l}\mathrm{p}- \\
\text { value }\end{array}$ \\
\hline No. of patients & 51 & 66 & & & & \\
\hline \multicolumn{7}{|l|}{ Age $\geq 70$ yrs } \\
\hline Yes & $\begin{array}{l}41 \\
(80.4 \%)\end{array}$ & $\begin{array}{l}63 \\
(95.5 \%)\end{array}$ & $\begin{array}{l}0.20 \\
(0.05- \\
0.75)\end{array}$ & 0.0157 & $\begin{array}{l}7.25 \\
(1.65- \\
43.01)\end{array}$ & 0.0076 \\
\hline \multicolumn{7}{|l|}{ Sex } \\
\hline Female & $\begin{array}{l}18 \\
(35.3 \%)\end{array}$ & $\begin{array}{l}28 \\
(42.4 \%)\end{array}$ & $\begin{array}{l}1.35 \\
(0.64- \\
2.87)\end{array}$ & 0.4524 & & \\
\hline \multicolumn{7}{|l|}{ Antiplatelet therapy } \\
\hline No & $\begin{array}{l}10 \\
(19.6 \%)\end{array}$ & $\begin{array}{l}28 \\
(42.4 \%)\end{array}$ & $\begin{array}{l}3.02 \\
(1.30- \\
7.04)\end{array}$ & 0.0101 & $\begin{array}{l}2.73 \\
(1.03- \\
7.87)\end{array}$ & 0.0440 \\
\hline \multicolumn{7}{|l|}{ Anticoagulant therapy } \\
\hline Yes & $\begin{array}{l}19 \\
(37.3 \%)\end{array}$ & $\begin{array}{l}36 \\
(54.6 \%)\end{array}$ & $\begin{array}{l}0.50 \\
(0.24- \\
1.04)\end{array}$ & 0.0923 & & \\
\hline \multicolumn{7}{|l|}{ Intracerebral hemorrhage } \\
\hline$\Gamma^{\prime}$ & $\begin{array}{l}12 \\
(23.5 \%)\end{array}$ & $\begin{array}{l}30 \\
(45.5 \%)\end{array}$ & $\begin{array}{l}0.37 \\
(0.17- \\
0.83)\end{array}$ & 0.0194 & $\begin{array}{l}1.73 \\
(0.60- \\
4.99)\end{array}$ & 0.3060 \\
\hline \multicolumn{7}{|l|}{$\begin{array}{l}\text { Enlargement of intracranial } \\
\text { hematoma }<72 \mathrm{hrs}\end{array}$} \\
\hline Yes & $8(16.0 \%)$ & $\begin{array}{l}27 \\
(46.6 \%)\end{array}$ & $\begin{array}{l}0.22 \\
(0.09- \\
0.55)\end{array}$ & 0.0009 & $\begin{array}{l}3.11 \\
(1.03- \\
10.20)\end{array}$ & 0.0447 \\
\hline \multicolumn{7}{|l|}{ Extracranial injury } \\
\hline Yes & $9(17.7 \%)$ & $9(13.6 \%)$ & $\begin{array}{l}1.36 \\
(0.50- \\
3.71)\end{array}$ & 0.6106 & & \\
\hline
\end{tabular}

Table 3 Comparison of the clinical outcomes of 117 head trauma patients that were taking antithrombotic drugs 95\%CI: 95\% confidence interval, IQR: interquartile range, mRS: mRS modified Rankin scale score, OR: odds ratio 